\title{
RNA-Seq Analyses of the Role of miR-21 in Acute Pancreatitis
}

\author{
Xun Lia Zhanwen Lin ${ }^{a} \quad$ Lei Wang $^{a}$ Qin Liu ${ }^{a}$ Zhi Cao ${ }^{b}$ Zhujuan Huang

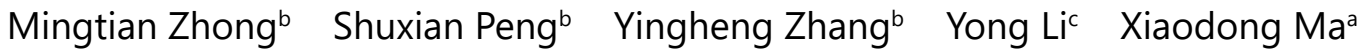 \\ alnstitute for Brain Research and Rehabilitation, Guangdong Key Laboratory of Mental Health and \\ Cognitive Science, Center for Studies of Psychological Application, South China Normal University, \\ Guangzhou, 'bThe Research Center of Basic Integrative Medicine, Guangzhou University of Chinese \\ Medicine, Guangzhou, China, 'Department of Cancer Biology, Lerner Research Institute, Cleveland \\ Clinic, Cleveland, USA
}

\section{Key Words}

miR-21 • RNA-sequencing • Acute pancreatitis $\cdot \mathrm{Hmgb} 1 \cdot$ Stat3 $•$ Pias3

\begin{abstract}
Background/Aims: Our previous study demonstrated that a deficiency of microRNA 21 (miR21) protects mice from acute pancreatitis, yet the underlying molecular networks associated with miR-21 in pancreatitis and pancreatitis-associated lung injury remain unexplored. Methods: We used next generation sequencing to analyze gene expression profiles of pancreatic tissues from wild-type (WT) and miR-21 knockout (KO) mice treated with caerulein by using a 1-day treatment protocol. The Database for Annotation, Visualization, and Integrated Discovery gene annotation tool and Ingenuity Pathway Analysis were used to analyze the molecular pathways, while quantitative real-time PCR, western blotting, and immunohistochemistry were used to explore the molecular mechanisms. Results: We identified 152 differentially expressed genes (DEGs) in pancreata between WT and KO mice treated with caerulein. Cellular biogenesis and metabolism were the major pathways affected between WT and KO mice, whereas cell death and inflammatory response discriminated between WT and KO mice under acute pancreatitis. We validated 16 DEGs, consisting of 6 upregulated genes and 10 downregulated genes, involved in pancreatic injury. In particular, the upregulation of Pias3 and downregulation of Hmgb1 in KO pancreata coincided with a reduced severity of pancreatitis. In addition, we found Hmgb1 stimulation resulted in the overexpression of miR-21 in peripheral blood mononuclear cells, and deletion of miR-21 led to a reduction of caerulein-induced acute pancreatitis-associated lung injury by repressing Hmgb1 expression. Conclusion: Our data support the hypothesis that miR-21 modulates the inflammatory response during acute pancreatitis through the upregulation of Pias 3 and downregulation of Hmgb1. Our findings further underscore a role for miR-21 in the promotion of acute pancreatitis.

X. Li, Z. Lin and L. Wang contributed equally to this work.

Xiaodong Ma

KARGER
Institute for Brain Research and Rehabilitation, South China Normal University No. 55, West of Zhongshan Avenue, 510631 Guangzhou (China)

Tel. +86 02015626217025, Fax 86-020-85216631, E-Mail sciencema@hotmail.com 


\section{Cellular Physiology Cell Physiol Biochem 2018;51:2198-2211

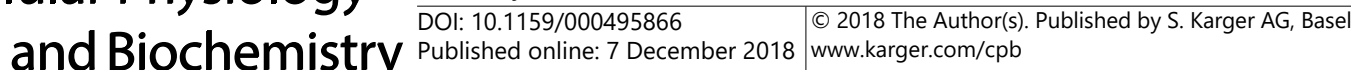

Li et al.: miR-21 Targeting Pias3 and Hmgb1 in Pancreatitis

\section{Introduction}

Acute pancreatitis is a common disease that frequently involves pathobiological processes such as inflammation, edema, and necrosis of pancreatic acinar cells, often accompanied by complications such as liver and lung injury [1-3]. In severe cases of acute pancreatitis, the release of inflammatory cytokines can cause systemic inflammatory response syndrome, which is associated with dysfunction of vital end organs. Multiple organ failure, the main cause of death, occurs in $10-15 \%$ of patients with acute pancreatitis [4]. The initial pathological change of acute pancreatitis is injury to pancreatic acinar cells caused by the uncontrolled intra-pancreatic activation of proteases, resulting in the accumulation of inflammatory cells and cytokines and the subsequent onset of a systemic cascade reaction. Therefore, the severity of acute pancreatitis is dependent on the mechanism of cell death of pancreatic acinar cells and inflammatory injury.

Recently, significant progress has been achieved in understanding cell death in acute pancreatitis. Two types of cell death, apoptosis and necrosis, are found during severe acute pancreatitis. Apoptosis, displaying chromatin aggregation, nuclear condensation, and chromosomal DNA fragmentation, is executed by caspases orchestrating cellular destruction with proteolytic cascades [5]. Necrosis, exhibiting cytoplasmic swelling and disruption of the plasma membrane, is positively correlated with the severity of acute pancreatitis [6]. Milder forms of experimental pancreatitis in rats induced by supramaximal doses of caerulein are characterized by high numbers of apoptotic cells and low numbers of necroptotic cells $[7,8]$. In contrast, significantly more caerulein injections are required to produce acute pancreatitis in mice compared with rats. In addition, caerulein injections in mice result in fewer apoptotic cells and significantly more necroptotic cells, which are correlated with the severity of pancreatic injury. These observations support the hypothesis that the progression of pancreatitis is different between mice and rats.

Although it is unclear whether apoptosis or necrosis is a better representative of the initiation and progression of clinical acute pancreatitis, there is a general consensus that strategies aimed at inhibiting necrosis would improve outcome in patients with severe pancreatitis. Thus, the identification of potential therapeutic targets to inhibit necrosis or "switch" necrosis to apoptosis during acute pancreatitis could improve outcome.

Among the many different forms of regulated necrosis, necrotic cell death that depends on receptor-interacting protein 3 (RIP3, also known as RIPK3) is termed necroptosis, which is a cellular process integrated with the extrinsic apoptosis pathway and called regulated necrosis. Extrinsic apoptosis is regulated by death receptors, such as tumor necrosis factor receptor (TNFR), and the TNFR-mediated initiation of necroptosis requires the kinase activity of RIP1 (also known as RIPK1) and RIP3 [9-11]. Both RIP1 and RIP3 are components of the necrosome, and caspase-8 can directly cleave and inactivate both kinases to negatively regulate necroptosis induced by TNF $\alpha$. Necroptosis is associated with several pathologic conditions, including acute pancreatitis, ischemia-reperfusion injury, neurodegeneration, and infection [9]. In addition, RIP3-dependent programmed necrosis (necroptosis) is found in necrotic acinar cells in mouse models of acute pancreatitis [10]. Pharmacological inhibition of RIP3 and its downstream activator protein, mixed lineage kinase domain-like protein, reduces the severity of pancreatitis, thereby lessening the burden of systemic inflammatory response syndrome and associated organ injury $[12,13]$. Thus, manipulating the cell death mechanisms that involve caspase and RIP activation and driving necroptosis to apoptosis are novel therapeutic strategies against acute pancreatitis.

MicroRNA 21 (miR-21) is an "oncomiR" that promotes oncogenesis mainly by inhibiting cellular apoptosis [14]. Our previous work showed that deletion of miR-21 protects mice from acute pancreatitis induced by two series of 8-hourly caerulein intraperitoneal (i. p.) injections by modulating necroptosis [15]. Our previous results demonstrated that caeruleininduced acute pancreatitis in wild-type (WT) mice receiving miR-21 knockout (KO) bone marrow was similar to that observed in WT mice transplanted with WT bone marrow, which excludes the possibility that inflammatory infiltration by macrophages or other leukocytes 
plays an essential role in the less severe acute pancreatitis phenotype in miR-21 KO mice. Although inflammatory cell infiltration is not the main reason for the reduction of pancreatitis in miR-21 KO mice, reduced injury to distal organs such as the lung was found in miR-21 KO mice compared with WT mice treated with caerulein. Thus, the molecular mechanism of the inflammatory response involved in miR-21 and acute pancreatitis and organ failure remains unclear. In the present study, we used a 1-day protocol of 8-hourly caerulein i. p. injections and further analyzed the molecular mechanisms of cell death and inflammatory responses involved in the protective role of miR-21 deficiency in acute pancreatitis and its associated lung injury.

\section{Materials and Methods}

\section{Acute pancreatitis models}

The generation of miR-21 KO mice on a C57BL/6X129S hybrid background has been described previously [14], and these mice were backcrossed with C57BL/6J mice for 10 generations and donated to the Jackson Laboratory (reference: Mir21 ${ }^{\text {tm1Yoli }}$. Female WT and KO mice (20-25 g, 8-10 weeks old) were used for caerulein-induced acute pancreatitis. The mice were administered 8-hourly i.p. injections of $50 \mu \mathrm{g} / \mathrm{kg}$ caerulein (Sigma, St. Louis, MO). The mice were sacrificed at $24 \mathrm{~h}$ after the first caerulein injection and pancreatic tissues and serum were obtained. No lethality was observed. All mice were kept under specific pathogen-free conditions, and all mouse experiments were performed under a protocol (K2017002) approved by the Southern Medical University Institutional Animal Care and Use Committee and in accordance with the Guide for the Care and Use of Laboratory Animals (National Institutes of Health, Bethesda, MD). Quantification of pancreatic injury was evaluated based on hematoxylin and eosin staining.

\section{Enzyme activity}

WT and KO mice were administered 8-hourly i. p. injections of caerulein, and they were sacrificed at the indicated time point. Blood was collected through the tail vein and kept at $4^{\circ} \mathrm{C}$ for $1 \mathrm{~h}$ before centrifugation at $4000 \times g$ for $20 \mathrm{~min}$ at $4^{\circ} \mathrm{C}$. Serum was collected from the supernatant. Serum amylase activity was measured by using an Amylase Assay Kit (Abcam, Cambridge, UK), and the serum level of Hmgb1 was measured by using an HMGB1 ELISA Kit (Shino-Test Corporation, Tokyo, Japan) according to the manufacturer's protocol. For MPO activity [15], lung samples were homogenized in $20 \mathrm{mM}$ phosphate buffer (pH 7.4) and the pellet was resuspended in $50 \mathrm{mM}$ phosphate buffer ( $\mathrm{pH} 6.0$ ) containing $0.5 \%$ hexadecyltrimethylammonium bromide (Sigma-Aldrich). The suspension was sonicated for $30 \mathrm{~s}$ and centrifuged at $10000 \times \mathrm{g}$ for $5 \mathrm{~min}$, and the supernatant was used for the MPO assay. The supernatant was mixed with $1.6 \mathrm{mM}$ tetramethylbenzidine (Sigma-Aldrich), $80 \mathrm{mM}$ sodium phosphate buffer ( $\mathrm{pH}$ 5.4), and $0.3 \mathrm{mM}$ hydrogen peroxide. This mixture was then incubated at $37^{\circ} \mathrm{C}$ for $110 \mathrm{~s}$, the reaction was terminated with $0.18 \mathrm{M} \mathrm{H}_{2} \mathrm{SO}_{4}$, and absorbance was measured at $450 \mathrm{~nm}$.

\section{RNA preparation and sequencing}

WT and KO mice were administered 8-hourly i. p. injections of caerulein. The mice were sacrificed at $24 \mathrm{~h}$ after the first caerulein injection, and pancreatic tissue was harvested at 1 day after the last caerulein injection. Total RNA was extracted using the TRI Reagent and a Direct-zol ${ }^{\mathrm{TM}}$ RNA Kit (Zymo Research, Irvine, CA) following the manufacturer's instructions. RNA quality and quantity were assessed on an Agilent Bioanalyzer 2100 (Agilent Technologies, Santa Clara, CA) to ensure an A260/A280 ratio in the range of 1.82.0 and an rRNA ratio $(28 \mathrm{~S} / 18 \mathrm{~S})>1.0$. DNA was removed by DNase I. Poly (A) RNAs were purified by using a Dynabeads mRNA Purification Kit (Invitrogen, Carlsbad, CA) according to the manufacturer's instructions. Poly (A) RNAs were fragmented at $94^{\circ} \mathrm{C}$ for $5 \mathrm{~min}$ before being reverse transcribed to cDNA with random primers. cDNA fragments were blunted and ligated to sequencing adaptors. Libraries were sequenced with the High-Seq 2000 Illumina sequencing platform by a sequencing service company (Cofactor Genomics, San Francisco, CA). 


\begin{tabular}{|c|c|c|}
\hline Callula & Cell Physiol Biochem 2018;51 & 1:2198-2211 \\
\hline ind Biochemistry & $\begin{array}{l}\text { DOl: 10.1159/000495866 } \\
\text { Publisned online: / December } 2018\end{array}$ & $\begin{array}{l}\text { (e } 2018 \text { The Author(s). Published by S. Karger AG, Basel } \\
\text { www.karger.com/cpb }\end{array}$ \\
\hline
\end{tabular}

Li et al.: miR-21 Targeting Pias3 and Hmgb1 in Pancreatitis

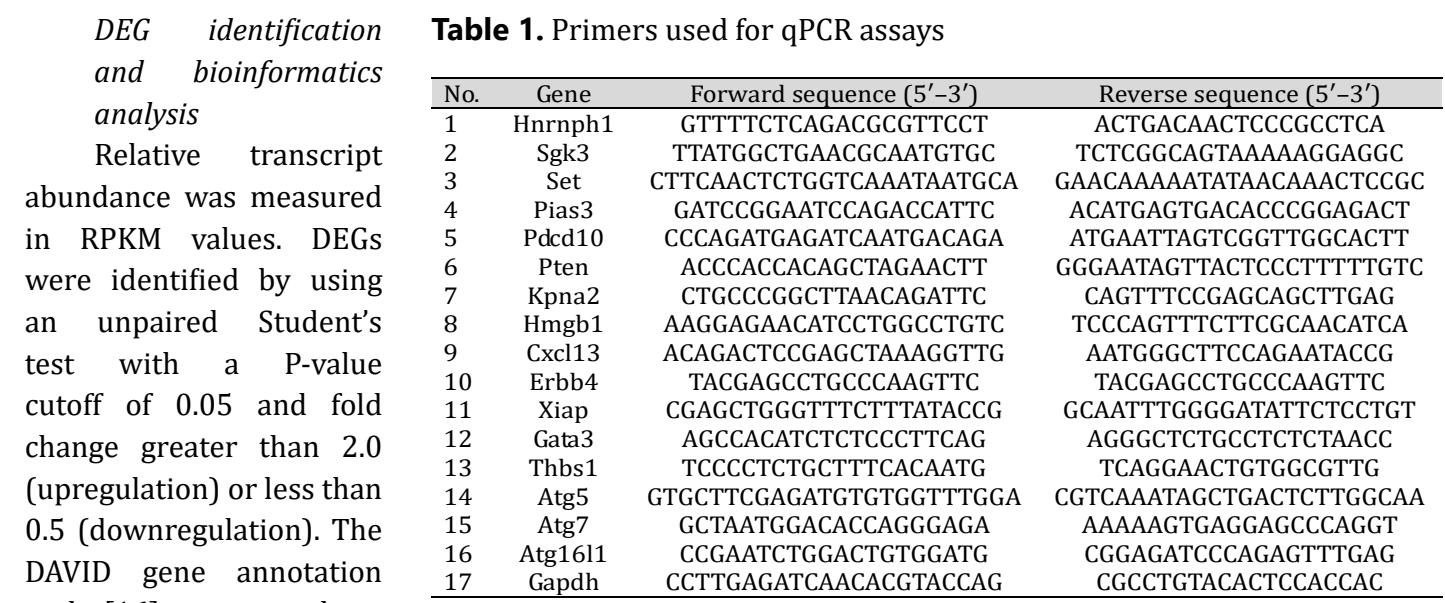

tool [16] was used to analyze the function of

DEGs according to GO with a significance of P-value $<0.05$ and FDR $<0.05$. IPA was used to identify enriched molecular pathways, gene networks, upstream regulators, canonical pathways, and cellular biological consequences.

qPCR

To validate gene expression, pancreatic tissues were dissected from the mice, and total RNA was extracted using TRIzol (Invitrogen) and then reverse transcribed with an iScript Reverse Transcription Supermix Kit (Bio-Rad Laboratories, Hercules, CA). qPCR was performed using SYBR Green Supermix (BioRad Laboratories) and a CFX96 Real-time PCR detection system (Bio-Rad Laboratories). The primers (Table 1) used for qPCR were designed based on the Universal ProbeLibrary (Roche Life Science, Pleasanton, CA). We analyzed each sample in duplicate with Gapdh as a reference. Real-time PCR for miR-21 detection was performed using a TaqMan assay (Thermo Fisher Scientific, Waltham, MA).

\section{Injection of plasmid DNA}

Lentivirus-based shRNA vectors were constructed as described previously [17]. The sequence of the Pias3 shRNA oligonucleotides was: PIAS3, 5'-GGGCACATTGGGCAGTGATTT-3'; Scramble (shRNA control), 5'-GTGCCGGAAGGCGTATATGTT-3'. The shRNA-expression lentiviral vectors were generated by cloning gene-specific or scramble shRNA sequences into pSIF-copGFP vectors. Plasmid DNA was prepared using an EndoFree plasmid system (Zymo Research) according to the manufacturer's instructions. Hydrodynamic injection of plasmid DNA was performed as described previously $[18,19]$. In brief, $50 \mu \mathrm{g}$ plasmid DNA was injected i.p. at $36 \mathrm{~h}$ before the first caerulein injection. The effect of shRNA on PIAS3 protein expression was examined using western blot analysis.

\section{PBMC isolation and treatment}

PBMCs were isolated from WT and miR-21 KO mice using the Ficoll-Paque method. Briefly, blood samples from WT and miR-21 KO mice were isolated and diluted $2 \times$ with phosphate-buffered saline plus $2 \%$ fetal bovine serum, and then added to the top of Ficoll solution in a 15-mL tube. The tubes were centrifuged at $400 \times g$ at room temperature for $30 \mathrm{~min}$. The upper plasma layer was removed and discarded, and the remaining plasma-Ficoll interface solution was collected. A total of $1.0 \times 10^{6}$ cells/well were seeded in 6 -well plates and treated with rHmgb1 (eBioscience, San Diego, CA) and/or an anti-Hmgb1-neutralizing antibody (Clone: 3E8; BioLegend, San Diego, CA) at the indicated concentrations and time points. PBMCs were also treated with TPA $(5 \mathrm{ng} / \mathrm{mL})$ and innomycin $(0.5 \mu \mathrm{mol} / \mathrm{L})$ at the indicated time points.

\section{Western blotting analysis}

Pancreatic tissues and cells were lysed with RIPA buffer (Cell Signaling Technology, Danvers, MA) supplemented with protease inhibitor (Thermo Fisher Scientific). The collected protein samples were boiled for $5 \mathrm{~min}$ at $95^{\circ} \mathrm{C}$. Protein concentration was measured with a bicinchoninic acid protein assay (Pierce, Rockford, IL). Equal amounts of protein were subjected to sodium dodecyl sulfate polyacrylamide 


\section{Cellular Physiology Cell Physiol Biochem 2018;51:2198-2211

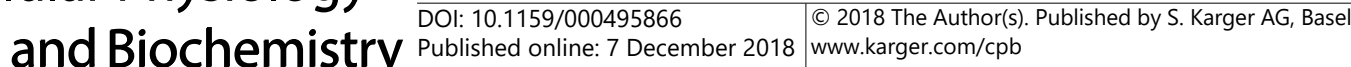 \\ Li et al.: miR-21 Targeting Pias3 and Hmgb1 in Pancreatitis}

-polyacrylamide gel electrophoresis before being transferred to a polyvinylidene difluoride membrane for blotting. The membrane was blocked with 5\% nonfat dry milk in Tris-buffered saline with Tween 20 buffer for $1 \mathrm{~h}$ and incubated with a primary antibody at $4^{\circ} \mathrm{C}$ overnight. Primary antibodies against Pias3 (4164), phospho-Stat3 (D3A7), and Stat3 (124H6) were purchased from Cell Signaling Technology, and antibodies against Hmgb1 (H9664) and $\beta$-actin (A2228) were purchased from Sigma-Aldrich. Horseradish peroxidaseconjugated anti-rabbit or anti-mouse IgG was used as a secondary antibody. Immunoreactive proteins were visualized with a West Dura Chemiluminescent Kit (Thermo Fisher Scientific). Quantitation of X-ray film was carried out by scanning densitometry using area integration.

\section{Histology and immunohistochemistry analyses}

Pancreatic or lung tissues were collected at the indicated times and perfused with $10 \%$ neutral buffered formalin. After fixation for $24 \mathrm{~h}$, the tissues were paraffin-embedded, sectioned $(4 \mu \mathrm{m})$, and stained with hematoxylin for pancreatic morphological analysis or hematoxylin and eosin for lung tissue analysis. For immunohistochemistry staining, deparaffinized and rehydrated sections were boiled in Na-citrate buffer (10 mM, pH 6.0) for $30 \mathrm{~min}$ for antigen retrieval. The sections were incubated with primary antibodies and developed by using the Ultra Vision Detection System (Thermo Fisher Scientific). Images were acquired using an Olympus IX51 microscope and processed using cellSens Dimension software.

\section{Statistical analysis}

Data are presented as the mean \pm standard deviation (SD). SPSS14.0 software (SPSS, Inc., Chicago, IL) was used for all statistical analyses. One-way analysis of variance was performed, and statistical significance was set at $* \mathrm{P}<0.05$ or $* * \mathrm{P}<0.01$.

\section{Data availability}

The data supporting the findings of this study were submitted to the Gene Expression Omnibus database (accession number: GSE106550; https://www.ncbi.nlm.nih.gov/geo/query/acc.cgi?acc=GSE106550).

\section{Results}

Response of WT and miR-21 KO mice treated with caerulein within 1 day

We adopted a new protocol using a 1-day protocol of 8-hourly caerulein injections to achieve acute pancreatitis (Fig. 1A). We first measured the expression of miR-21 in WT mice treated with caerulein by quantitative real-time PCR (qPCR). Consistent with previous studies, we found that, compared with saline-treated controls, the $\Delta \mathrm{Ct}$ values of miR-21 normalized to a reference RNA (snoRNA292) were significantly lower following caerulein treatment $(\mathrm{P}=0.005$; Fig. $1 \mathrm{~B})$, representing a $>3.2$-fold upregulation of miR-21 expression. Next, we evaluated the histological changes of pancreata from caerulein-treated WT and miR-21 KO mice. There was less acinar cell injury and necrosis in pancreatic tissues from KO mice compared with WT mice (Fig. 1C). Serum amylase, a biomarker of acute pancreatitis, was highly elevated in WT mice treated with caerulein compared with those treated with vehicle (saline). Nevertheless, there was significantly lower serum amylase activity in KO mice than in WT mice treated with caerulein at $1 \mathrm{~h}$ after the last injection (Fig. 1D). These data are in line with our previous reported results that deletion of miR-21 is protective against pancreatic injury in mice using 2 days of caerulein treatment to induce acute pancreatitis [15]. Therefore, we adopted this 1-day protocol in the following studies to identify differentially expressed genes (DEGs) in pancreatic tissues between WT and KO mice.

\section{Gene ontology and Ingenuity Pathway Analysis studies of DEGs}

We performed RNA-sequencing (RNA-seq) using total RNA isolated from the pancreatic tissues of WT and KO mice treated with caerulein. After quality filtering and normalizing the raw sequencing data, we identified DEGs based on the following criteria: fold change $>2$ or $<0.5$, P-value $<0.05$, and false discovery rate (FDR) $<0.05$. By comparing the reads per kilobase per million (RPKM) values between the two groups, a total of 152 DEGs (57 
upregulated and 95 downregulated) were identified in the pancreas of KO mice treated with caerulein (KO-Caerulein) compared with WT mice treated with caerulein (WT-Caerulein). In an effort to understand the molecular and cellular impacts of these DEGs, we analyzed both the upregulated and downregulated genes using Database for Annotation, Visualization, and

Fig. 1. miR-21 deficiency protects against acute pancreatitis in mice treated with a 1-day caerulein protocol. (A) Schematic representation of the caerulein treatment procedure. (B) Expression of miR-21 in pancreata from WT mice treated with caerulein within 1 day. (C) Representative histological sections of pancreata stained with hematoxylin. (D) Serum amylase activity from WT and miR-21 KO mice at $1 \mathrm{~h}$ after the last caerulein injection. Values represent the mean \pm SD (n

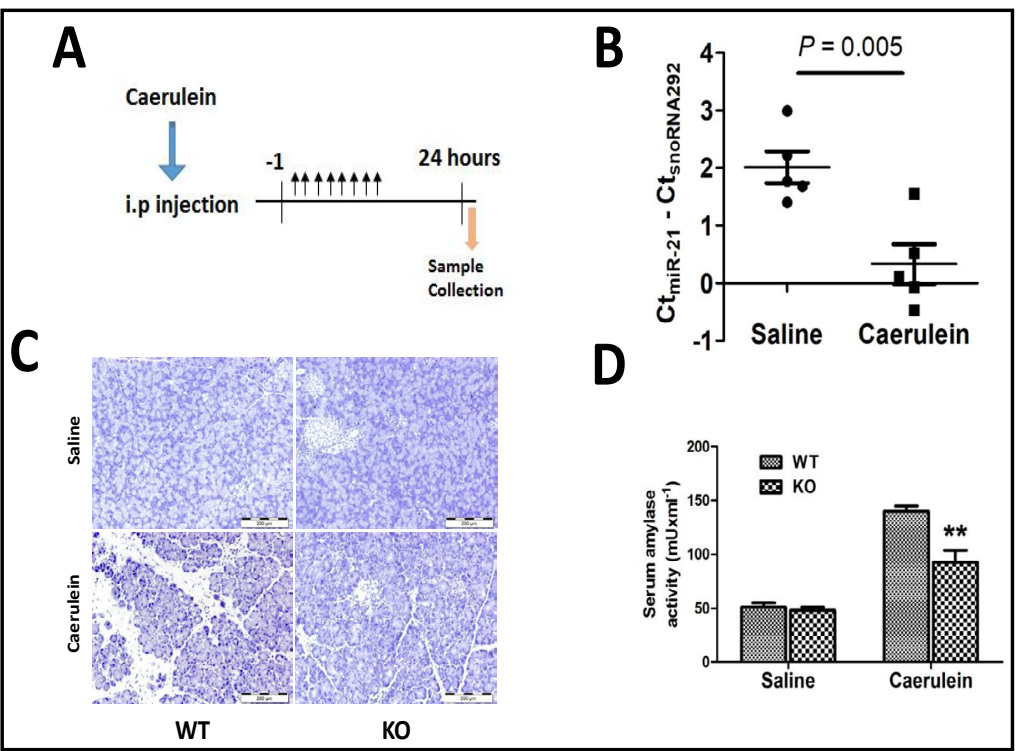

$=5) .{ }^{* *} \mathrm{P}<0.01$ indicates a significant difference between WT and miR-21 KO mice treated with caerulein.

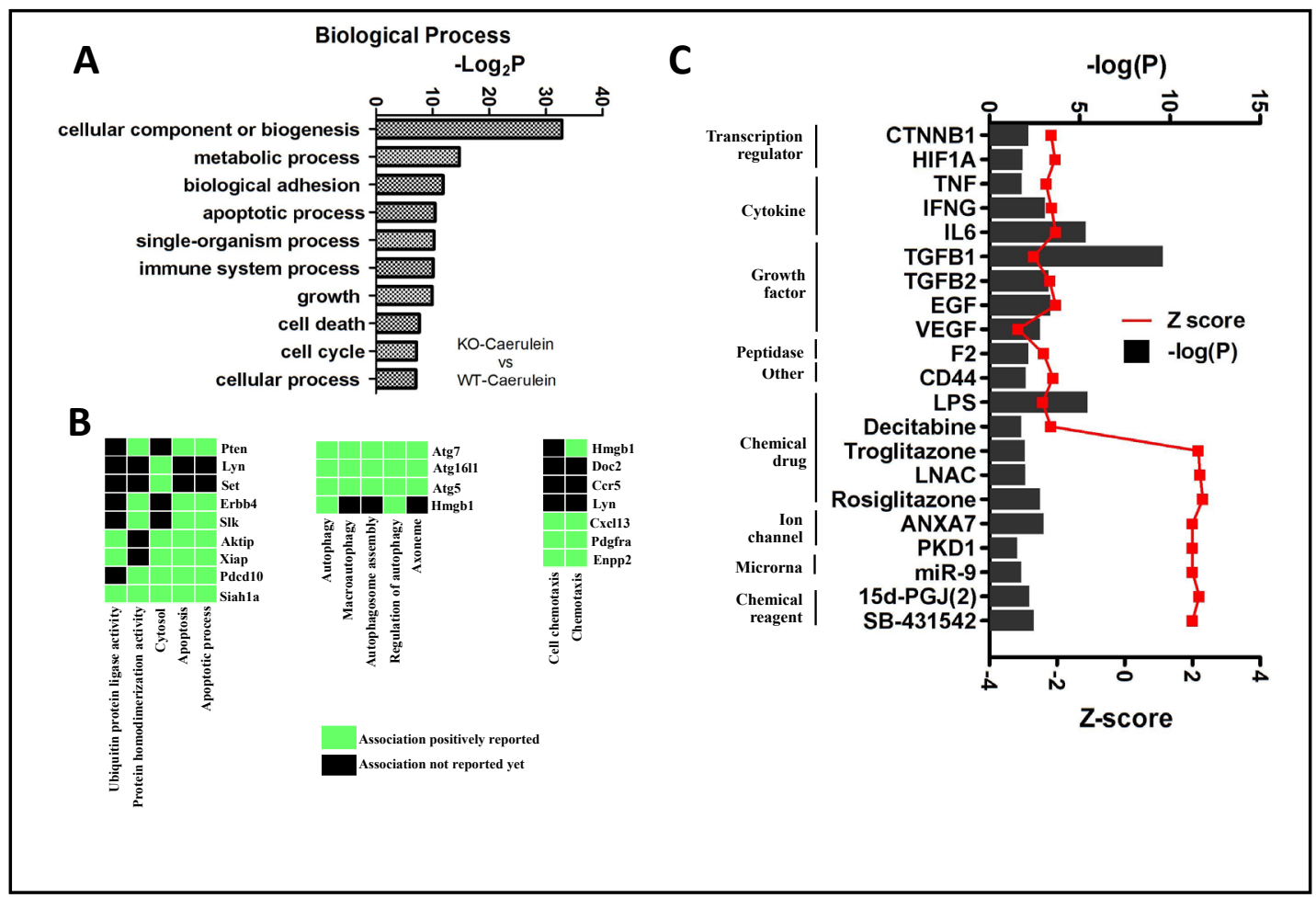

Fig. 2. GO and IPA studies of dysregulated genes between WT and KO mice treated with caerulein. (A) GO enrichment analysis of biological processes involving DEGs between WT-caerulein and KO-caerulein mice. (B) 2D view analysis showing apoptosis, autophagy, and chemotaxis genes between WT-caerulein and KOcaerulein mice. (C) Upstream pathway analysis (-logP, upper axis, black bars; activation Z score, lower axis, red) of the DEG signature. 
Integrated Discovery (DAVID) bioinformatics resources [16]. By analyzing the significant factors within the biological process domain, we found that cellular biogenesis and metabolic process were the most significant gene ontology (GO) terms between WT-Caerulein and KOCaerulein mice (Fig. 2A). Importantly, immune system process and cellular death were also found to be significant GO terms. Since miR-21 has been reported to promote necroptosis by regulating the intrinsic apoptosis pathway [15], we then focused on cell death as a key biological endpoint of acute pancreatitis in the following analyses. Functional annotation clustering was performed to show the DEGs in a two-dimensional (2D) display. Apoptosisassociated genes, including Erbb4, Slk, Aktip, Xiap, Pdcd10, and Siah1a, were identified by comparing WT-Caerulein mice with KO-Caerulein mice (Fig. 2B, left). Beyond apoptosis, autophagy, another regulated form of cell death, was also found to be closely correlated with acute pancreatitis due to miR-21 deficiency (Fig. 2B, middle). By clustering analysis using a 2D display format, four autophagy-related genes, namely, Atg5, Atg7, Atg16l1, and Hmgb1, were identified as DEGs between WT-Caerulein mice and KO-Caerulein mice, indicating a potential role for autophagy in miR-21-mediated acute pancreatitis. In addition, chemotaxisrelated genes, such as Hmgb1 and Cxcl13, were also involved in the protective role of miR-21 deletion in acute pancreatitis (Fig. 2B, right).

Next, we used Ingenuity Pathway Analysis (IPA) software in conjunction with the DEGs identified between WT-Caerulein and KO-Caerulein mice to explore the enriched molecular pathways. The activation Z-scores of transcriptional regulators, cytokines, growth factors, and peptidases were found to be inhibited, whereas most of the chemical drugs, ion channels, and chemical reagents were activated (Fig. 2C). The most implicated IPA network was "Cellular Assembly and Organization, Cellular Function and Maintenance." In addition, "Cell Death and Survival, Embryonic Development, Cell-To-Cell Signaling and Interaction" was among the top 5 IPA networks. IPA analysis found that the caspase, nuclear factor- $\kappa \mathrm{B}$, extracellular signal-regulated kinases $1 / 2$, interleukin-1, and transforming growth factor- $\beta$ signaling pathways were in the core modules during pancreatitis (Supplementary Fig. S1A - for all supplemental material see www.karger.com/10.1159/000495866/). Specifically, 58 DEGs were shown to be associated with a cell death signaling network consisting of several upregulated genes involved in apoptosis, including Pten, Pias3, Set, and Pdcd10, and downregulated genes involved in necrosis, such as Hmgb1, which were also connected to chemotaxis and inflammatory response (Supplementary Fig. S1B and S1C). Taken together, our data suggest that the cell death and inflammatory response pathways may be involved in the differences between WT and KO mice under acute pancreatitis.

\section{qPCR validation of the upregulated and downregulated DEGs}

Next, we selected cell death and inflammatory response-associated genes to validate the RNA-seq data using qPCR. We selected 6 upregulated genes (Hnrnph1, Sgk3, Set, Pias3, Pdcd10, and Pten) and 10 downregulated genes (Kpna2, Hmgb1, Cxcl13, Erbb4, Xiap, Gata3, Thbs1, Atg5, Atg7, and Atg16l1) in K0-Caerulein mice compared with WT-Caerulein mice to perform qPCR (Table 1). The 6 upregulated genes were validated by qPCR, but showed no difference in expression between WT-Saline and KO-Saline mice (Fig. 3A). Among these 6 genes, Pten and Pias 3 are experimentally validated miR-21 target genes. As expected, the 10 significantly downregulated genes were also validated by qPCR (Fig. 3B).

\section{Pias3 and Stat3 are involved in the protective role of miR-21 deletion in caerulein-induced} pancreatitis

We explored the molecular pathways that were enriched for the 16 validated DEGs using IPA. Network analysis indicated that the Stat3, Pten, XIAP, and p53 signaling pathways were the core modules regulated by these DEGs (Supplementary Fig. S2A). Pias3, a miR-21 target gene, inhibits Stat 3 activation and participates in acute pancreatitis [20,21]. First, we determined whether miR-21 deletion affected Pias3 expression and the activation of Stat3 by western blotting. Pias3 expression was not significantly different in pancreatic tissue of WT mice treated with saline compared with KO mice treated with saline (Fig. 4A). As shown 
Fig. 3. $q P C R$ validation of the upregulated and downregulated DEGs. WT and KO mice were treated with saline (WT-Saline, WT-S; KO-Saline, KO-S) or caerulein (WT-Caerulein, WT-C; KOCaerulein, KO-C). The mice were sacrificed at $24 \mathrm{~h}$ after the first saline or caerulein injection and pancreatic tissues were obtained (5 mice per group). Upregulated (A) and downregulated (B) DEGs were validated by qPCR. Values represent the mean \pm SD of three independent experiments. ${ }^{* *} \mathrm{P}<$ 0.01 .

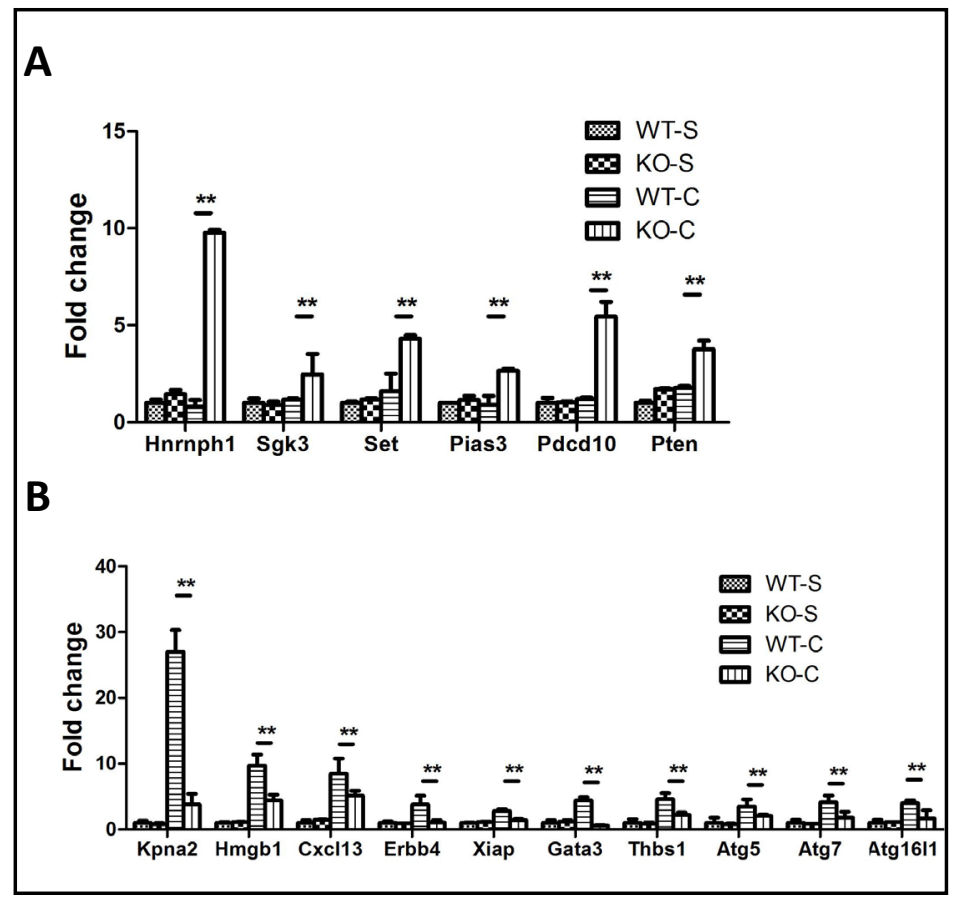

Fig. 4. miR-21 deletion protects mice from c a e rulein - in d u ced pancreatitis by elevating Pias3 expression. (A and B) Western blotting analyses of Pias3, phospho-Stat3, and total Stat3 levels in pancreata from mice. Pancreata of WT and KO mice treated with saline or caerulein were harvested at 24 $\mathrm{h}$ after the first dose (1 animal sample per lane). (C) Western blotting analyses of Pias3, phospho-Stat3, and total Stat3 levels in pancreata from KO mice injected with shRNAcontrol (shRNA-CTL) or shRNA-Pias3 prior to caerulein treatment. (D) Representative

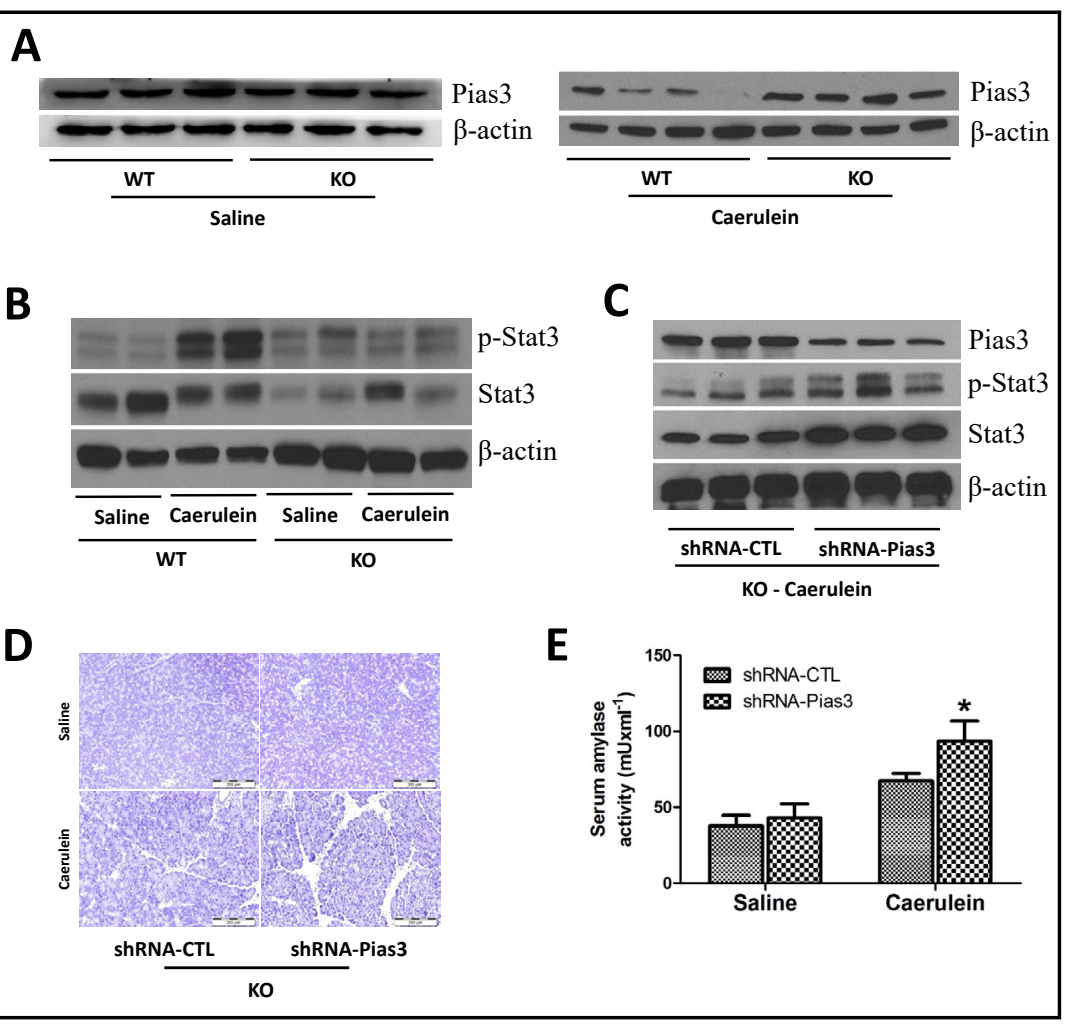
histological sections of

KO mice injected with shRNA-control (shRNA-CTL) or shRNA-Pias3 prior to saline or caerulein treatment. (E) Serum amylase activity in KO mice at $6 \mathrm{~h}$ after the last injection. Values represent the mean $\pm \operatorname{SD}(n=5)$. $* \mathrm{P}<0.05$ indicates a significant difference between shRNA-CTL and shRNA-Pias3 treatment. 
Fig. 5. Hmgb1 induces the expression of miR-21, and deletion of miR-21 inhibits Hmgb1 expression in PBMCs. (A) PBMCs were isolated from WT mice, and miR21 expression was measured after stimulation with 0 , $0.5,1$, or $2 \mu \mathrm{g} / \mathrm{mL}$ rHmgb1. (B) miR-21 expression in PBMCs was measured at 0 , 1,6 , and $24 \mathrm{~h}$ after stimulation with $1 \mu \mathrm{g} / \mathrm{mL}$ rHmgb1. (C) PBMCs were co-stimulated with rHmgb1 (1 $\mu \mathrm{g} / \mathrm{mL})$

and an anti-Hmgb1-neutralizing antibody $(1 \mu \mathrm{g} / \mathrm{mL})$ or IgG antibody control, and miR-21 expression was measured after $24 \mathrm{~h}$. (D) PBMCs were treated with TPA ( $5 \mathrm{ng} / \mathrm{mL})$ and innomycin $(0.5 \mu \mathrm{mol} / \mathrm{L})$ at 0,6 , and $24 \mathrm{~h}$, and proteins were extracted. Western blotting was performed to analyze the expression of Hmgb1 protein in PBMCs. ${ }^{*} \mathrm{P}<0.05$ and ${ }^{* *} \mathrm{P}<0.01$ indicate a significant difference between the indicated groups.

in Fig. 4A and 4B, Pias3 expression was increased, whereas Stat3 activation was markedly inhibited in the pancreas of KO mice compared with WT mice treated with caerulein. To determine further whether increased Pias3 expression is responsible for the protective effects of miR-21 deficiency against caerulein-induced acute pancreatitis, we pre-treated the mice with short hairpin RNA targeting Pias3 (shRNA-Pias3). shRNA-Pias3 inhibited Pias3 expression and led to the activation of Stat3 in KO mice treated with caerulein (Fig. 4C). shRNA-Pias3 pre-treatment significantly enhanced the severity of pancreatitis in KO mice as judged by morphology and serum amylase levels (Fig. 4D and 4E). These results indicate that the genetic deletion of miR-21 protects against pancreatic injury during acute pancreatitis by modulating the Pias3/Stat3 pathway.

Hmgb1 induces miR-21 expression, and deletion of miR-21 inhibits Hmgb1 expression in peripheral blood mononuclear cells

Hmgb1, a nuclear damage-associated molecular pattern protein, coordinates the processes of inflammation, immunity, and cell death [22-24]. Our previous network analysis revealed that Hmgb1 was a key element in $\mathrm{KO}$ mice during acute pancreatitis (Supplementary Fig. 1B and 1C). Recently, Hmgb1 was reported to stimulate miR-21 expression in hepatocellular carcinoma cell lines [25]. We first determined whether Hmgb1 could regulate miR-21 expression in peripheral blood mononuclear cells (PBMCs). PBMCs were isolated from WT mice and treated with varying concentrations of recombinant mouse Hmgb1 (rHmgb1). Treatment of PBMCs with Hmgb1 resulted in a dose- and time-dependent increase of miR-21 expression (Fig. 5A and 5B, respectively). We next determined whether neutralizing Hmgb1 could prevent miR-21 expression. As expected, inhibiting Hmgb1 by using an anti-Hmgb1-neutralizing antibody significantly prevented miR-21 expression in PBMCs (Fig. 5C). Furthermore, we measured the expression level of Hmgb1 in PBMCs stimulated with TPA and innomycin. Hmgb1 was highly expressed in WT PBMCs at $24 \mathrm{~h}$ after stimulation with TPA and innomycin (Fig. 5D). More importantly, miR-21 KO resulted in decreased Hmgb1 expression in PBMCs, suggesting that the pancreatitis-associated injury to 


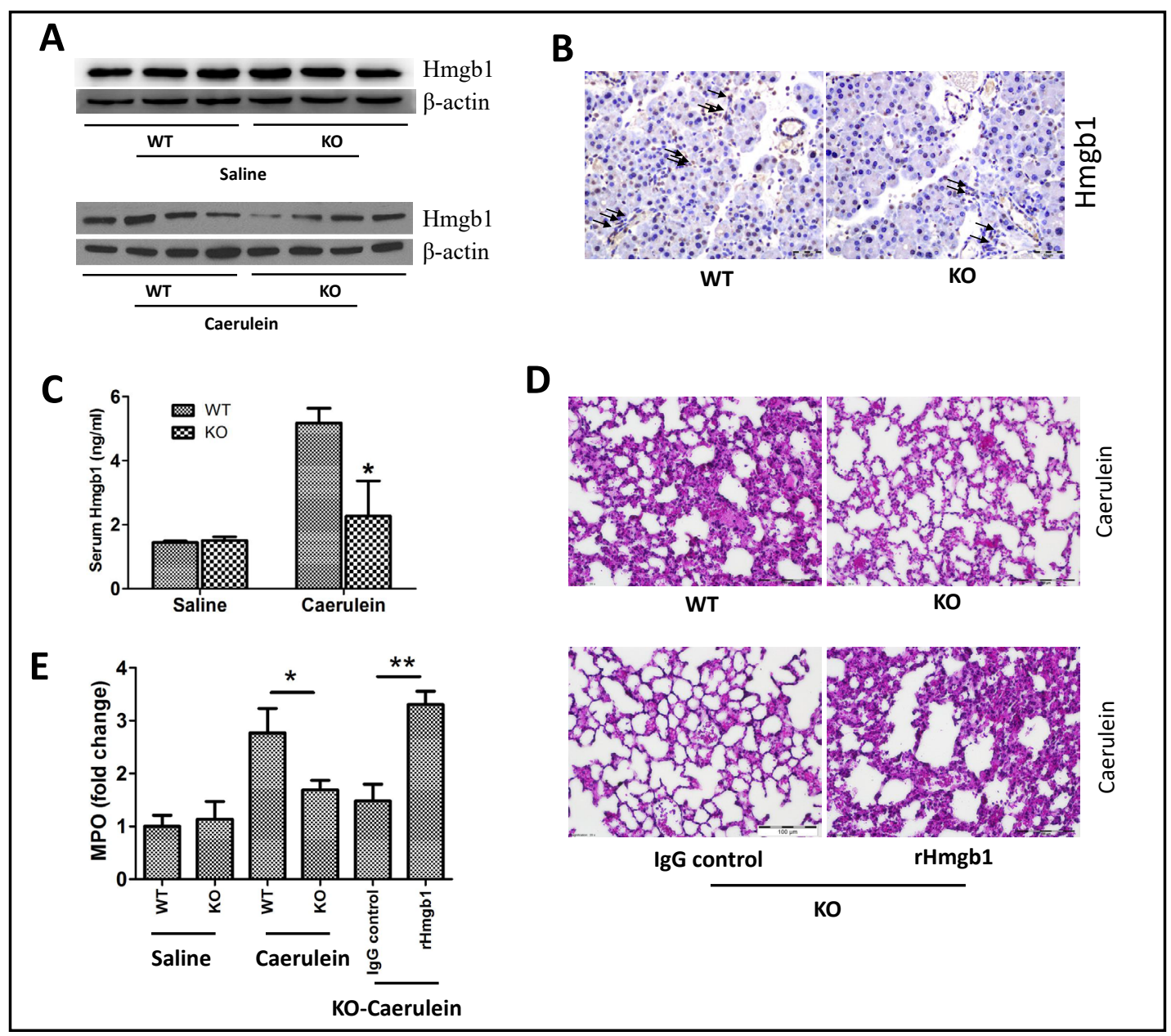

Fig. 6. miR-21 KO protects against pancreatitis-associated lung injury by downregulating Hmgb1 expression. (A) Western blotting analyses of Hmgb1 expression in the pancreas. Pancreata of WT and KO mice treated with saline or caerulein were harvested at $24 \mathrm{~h}$ after the first dose ( 1 animal sample per lane). (B) Immunohistochemistry analyses of Hmgb1 in the pancreas. Pancreata of WT and KO mice treated with caerulein were harvested at $24 \mathrm{~h}$ after the first dose. Pancreatic sections were stained with anti-Hmgb1 antibodies. Scale bars $=50 \mu \mathrm{m}$. Black arrows indicate Hmgb1-positive infiltrating immune cells. (C) Serum Hmgb1 levels from WT and KO mice at $1 \mathrm{~h}$ after the last caerulein or saline injection. (D) Representative histological sections of lung tissue stained with hematoxylin and eosin from caerulein-treated WT and KO mice (upper) or from caerulein-treated KO mice with rHmgb1 (or IgG control) injection (lower). (E) MPO activity was measured as described in the Materials and Methods. Values represent the mean \pm SD $(n=5)$. ${ }^{*} \mathrm{P}<0.05$ and ${ }^{* *} \mathrm{P}<0.01$ indicate a significant difference between the indicated groups.

distal organs may be reduced in KO mice and possibly due to the downregulation of Hmgb1.

Deletion of miR-21 protects mice from pancreatitis-associated lung injury by downregulating Hmgb1

To study the role of Hmgb1 in the protective effect of miR-21 deletion during acute pancreatitis, we first determined whether miR-21 deletion decreased the level of Hmgb1 protein by using western blotting and immunohistochemistry analyses. miR-21 deletion did not reduce the level of Hmgb1 protein in pancreatic tissue in mice treated with saline; however, the level of Hmgb1 protein was significantly downregulated in pancreatic tissue from KO mice compared with WT mice treated with caerulein (Fig. 6A and 6B). This result was confirmed by immunohistochemistry (Fig. 6B). Careful examination revealed that 
Hmgb1 expression was inhibited mostly in infiltrating immune cells (Fig. 6B, black arrows). Several studies have demonstrated that downregulation of Hmgb1 protects against damage to distal organs caused by acute pancreatitis, such as lung injury [26-29]. Therefore, we speculated that miR-21 ablation reduces the severity of lung injury during pancreatitis by downregulating Hmgb1 expression. We measured the serum level of Hmgb1 between WT and KO mice treated with caerulein. There was no significant difference in the serum level of Hmgb1 between WT and KO mice treated with saline, whereas it was significantly inhibited in KO mice compared with WT mice treated with caerulein (Fig. 6C). Lung injury, manifested as thickened alveolar membranes and myeloperoxidase (MPO) enzyme activity, was markedly lower in KO mice treated with caerulein compared with WT mice (Fig. 6D-upper and Fig. $6 \mathrm{E})$. When the level of Hmgb1 was restored by injecting rHmgb1 into KO mice treated with caerulein, the severity of lung injury was increased (Fig. 6D-lower and Fig. 6E). Collectively, these data suggest that the protective role of miR-21 deficiency in pancreatitis-associated lung injury is more likely due to the inhibition of Hmgb1 expression, and the downregulation of Hmgb1 is partially responsible for the reduced lung injury observed in mice lacking miR21.

\section{Discussion}

Genetic depletion of miR-21 in mice provides protection against pancreatic injury induced by the 2-day serial administration of caerulein [15], which reflects the regenerative capability of the pancreas [30]. In the current study, we demonstrated that miR-21 deficiency also protects mice from acute pancreatitis using a single day protocol of caerulein treatment. Our previous study identified two miR-21 targets (Pten and FasL) that were involved in regulating necrosis during acute pancreatitis, yet the underlying molecular networks associated with miR-21 in pancreatitis remain unexplored. Therefore, we performed RNA-seq to identify potential miR-21 targets and related signaling networks in acute pancreatitis. The major finding from this study was that deletion of miR-21 protected mice against pancreatitis by modulating the Pias3/Stat3 pathway and reduced lung injury by downregulating Hmgb1 expression.

We identified 57 upregulated genes and 95 downregulated genes in KO pancreata compared with WT controls treated with caerulein. As determined by GO analysis, the upregulated genes were primarily associated with metabolic process, whereas the downregulated genes were mostly related to cellular component biogenesis. Cell deathrelated genes were dysregulated in KO mice compared with WT mice treated with caerulein. These results demonstrated the critical role of cell death signaling pathways regulated by miR-21. Network analysis suggested that inflammatory response and cell death and survival are the top disease and biological functions associated with acute pancreatitis. Additionally, we found that autophagy-related genes, including atg5, atg7, atg1611, and Hmgb1, were downregulated in KO mice compared with WT mice treated with caerulein. Although the precise mechanism of autophagy in acute pancreatitis remains elusive, the current study implicates a significant role for autophagy in this process.

Inflammatory cells are essential for caerulein-induced pancreatic injury, and fewer inflammatory infiltrating cells are found in KO pancreata compared with WT control pancreata upon caerulein treatment [15]. According to the findings of bone marrow transplantation experiments, miR-21 deficiency in acinar cells, but not in inflammatory cells, protects mice from acute pancreatitis [15]. In the present study, genes associated with the inflammatory response, cell migration, and chemotaxis were significantly downregulated in KO mice compared with WT mice treated with caerulein. Pias3 is an inhibitor of Stat3, a core component of the Stat3 pathway that transactivates multiple key inflammatory cytokines. Pias3 is upregulated in pancreata from KO mice compared with WT mice, and Pias3 knockdown in pancreata of KO mice restored the severity of pancreatitis, suggesting that elevating Pias3 expression is a pivotal event mediated by miR-21 deficiency that protects

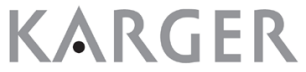




\section{Cellular Physiology Cell Physiol Biochem 2018;51:2198-2211

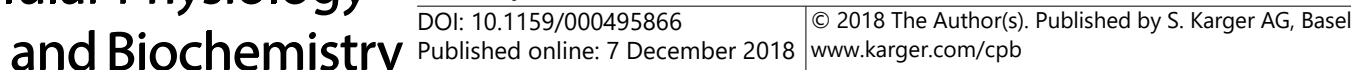 \\ Li et al.: miR-21 Targeting Pias3 and Hmgb1 in Pancreatitis}

mice from acute pancreatitis and could be a therapeutic target for acute pancreatitis.

Similar to the recent finding that Hmgb1 can stimulate miR-21 expression in hepatocellular carcinoma cell lines [25], our study revealed that Hmgb1 upregulated miR21 expression in PBMCs and the expression of Hmgb1 was significantly downregulated in both serum and pancreatic tissue from KO mice compared with WT mice treated with caerulein. In addition, the administration of rHmgb1 significantly decreased the inhibition of lung injury observed in KO mice treated with caerulein, which demonstrates that Hmgb1 and miR-21 participate in a common pathway linking inflammation to acute pancreatitis. Although it is unclear how miR-21 regulates Hmgb1 expression, these findings suggest that there are positive regulation and feedback loops between miR-21 and Hmgb1. As inhibiting the release of Hmgb1 protects against experimental acute pancreatitis and associated lung injury [31-33], the reduction of Hmgb1 in miR-21 KO mice helped to reduce lung injury.

\section{Conclusion}

Taken together, our data support the hypothesis that miR-21 modulates the inflammatory response during acute pancreatitis through the upregulation of Pias 3 and downregulation of Hmgb1. Data from this work support a key role for miR-21 in modulating the complex networks of cell death and the inflammatory response in acute pancreatitis. In future studies, it will be critical to determine how miR-21 enhances Hmgb1-mediated inflammatory and thereby creates a pro-inflammatory microenvironment with distant organ damage.

\section{Acknowledgements}

This study was supported by multiple grants to Xiaodong Ma from the Science and Technology Planning Project of Guangdong Province of China (No. 2014A050503062), the Natural Science Foundation of Guangdong Province of China (No 2017A030311004), the National Natural Science Foundation of China (Nos. 81572399 and 81773249), and the Science and Technology Program of Guangzhou City of China (No. 201707010139).

\section{Disclosure Statement}

All authors declare that they have no competing interests.

\section{References}

$>1$ Sand J, Nordback I: Acute pancreatitis: risk of recurrence and late consequences of the disease. Nature reviews Gastroenterology \& hepatology 2009;6:470-477.

$>2$ Peery AF, Crockett SD, Barritt AS, Dellon ES, Eluri S, Gangarosa LM, Jensen ET, Lund JL, Pasricha S, Runge T, Schmidt M, Shaheen NJ, Sandler RS: Burden of Gastrointestinal, Liver, and Pancreatic Diseases in the United States. Gastroenterology 2015;149:1731-1741 e1733.

-3 DiMagno MJ, DiMagno EP: New advances in acute pancreatitis. Current opinion in gastroenterology 2007;23:494-501.

-4 Forsmark CE, Vege SS, Wilcox CM: Acute Pancreatitis. The New England journal of medicine 2016;375:1972-1981.

5 Liu M, Shi L, Zou X, Zheng X, Zhang F, Ding X, Zhu H, Shen Y: Caspase inhibitor zVAD-fmk protects against acute pancreatitis-associated lung injury via inhibiting inflammation and apoptosis. Pancreatology 2016;16:733-738.

6 Wang G, Qu FZ, Li L, Lv JC, Sun B: Necroptosis: a potential, promising target and switch in acute 


\section{Cellular Physiology Cell Physiol Biochem 2018;51:2198-2211 \begin{tabular}{l|l|l} 
and Biochemistry Published onlıne: 7 December 2018 & $\begin{array}{l}\text { ○ } 2018 \text { The Author(s). Published by S. Karger AG, Basel } \\
\text { www.karger.com/cpb }\end{array}$ \\
\hline
\end{tabular}}

Li et al.: miR-21 Targeting Pias3 and Hmgb1 in Pancreatitis

pancreatitis. Apoptosis : an international journal on programmed cell death 2016;21:121-129.

7 Mareninova OA, Sung KF, Hong P, Lugea A, Pandol SJ, Gukovsky I, Gukovskaya AS: Cell death in pancreatitis: caspases protect from necrotizing pancreatitis. The Journal of biological chemistry 2006;281:3370-3381.

8 Bhatia M: Apoptosis versus necrosis in acute pancreatitis. American journal of physiology Gastrointestinal and liver physiology 2004;286:G189-196.

-9 Vandenabeele P, Galluzzi L, Vanden Berghe T, Kroemer G: Molecular mechanisms of necroptosis: an ordered cellular explosion. Nature reviews Molecular cell biology 2010;11:700-714.

10 He S, Wang L, Miao L, Wang T, Du F, Zhao L, Wang X: Receptor interacting protein kinase-3 determines cellular necrotic response to TNF-alpha. Cell 2009;137:1100-1111.

$\$ 11$ Han J, Zhong CQ, Zhang DW: Programmed necrosis: backup to and competitor with apoptosis in the immune system. Nature immunology 2011;12:1143-1149.

-12 Wu J, Huang Z, Ren J, Zhang Z, He P, Li Y, Ma J, Chen W, Zhang Y, Zhou X, Yang Z, Wu SQ Chen L, Han J: Mlkl knockout mice demonstrate the indispensable role of Mlkl in necroptosis. Cell research 2013;23:994-1006.

13 Wu J, Mulatibieke T, Ni J, Han X, Li B, Zeng Y, Wan R, Wang X, Hu G: Dichotomy between ReceptorInteracting Protein 1- and Receptor-Interacting Protein 3-Mediated Necroptosis in Experimental Pancreatitis. The American journal of pathology 2017;187:1035-1048.

14 Ma X, Kumar M, Choudhury SN, Becker Buscaglia LE, Barker JR, Kanakamedala K, Liu MF, Li Y: Loss of the miR-21 allele elevates the expression of its target genes and reduces tumorigenesis. Proceedings of the National Academy of Sciences of the United States of America 2011;108:10144-10149.

-15 Ma X, Conklin DJ, Li F, Dai Z, Hua X, Li Y, Xu-Monette ZY, Young KH, Xiong W, Wysoczynski M, Sithu SD, Srivastava S, Bhatnagar A: The oncogenic microRNA miR-21 promotes regulated necrosis in mice. Nature communications 2015;6:7151.

16 Huang da W, Sherman BT, Lempicki RA: Systematic and integrative analysis of large gene lists using DAVID bioinformatics resources. Nature protocols 2009;4:44-57.

17 Lu Z, Li Y, Takwi A, Li B, Zhang J, Conklin DJ, Young KH, Martin R: miR-301a as an NF-kappaB activator in pancreatic cancer cells. The EMBO journal 2011;30:57-67.

18 Uemura A, Takehara T, Miyagi T, Suzuki T, Tatsumi T, Ohkawa K, Kanto T, Hiramatsu N, Hayashi N: Natural killer cell is a major producer of interferon gamma that is critical for the IL-12-induced anti-tumor effect in mice. Cancer immunology, immunotherapy : CII 2010;59:453-463.

19 Shigekawa M, Hikita H, Kodama T, Shimizu S, Li W, Uemura A, Miyagi T, Hosui A, Kanto T, Hiramatsu N, Tatsumi T, Takeda K, Akira S, Takehara T: Pancreatic STAT3 protects mice against caerulein-induced pancreatitis via PAP1 induction. The American journal of pathology 2012;181:2105-2113.

-20 Xiong Q, Zhong Q, Zhang J, Yang M, Li C, Zheng P, Bi LJ, Ge F: Identification of novel miR-21 target proteins in multiple myeloma cells by quantitative proteomics. Journal of proteome research 2012;11:2078-2090.

-21 Zhang H, Neuhofer P, Song L, Rabe B, Lesina M, Kurkowski MU, Treiber M, Wartmann T, Regner S, Thorlacius H, Saur D, Weirich G, Yoshimura A, Halangk W, Mizgerd JP, Schmid RM, Rose-John S, Algul H: IL-6 trans-signaling promotes pancreatitis-associated lung injury and lethality. The Journal of clinical investigation 2013;123:1019-1031.

22 Scaffidi P, Misteli T, Bianchi ME: Release of chromatin protein HMGB1 by necrotic cells triggers inflammation. Nature 2002;418:191-195.

-23 Chen Z, Chen Y, Pan L, Li H, Tu J, Liu C, Dai X, Zhang X, Sun G, Feng D: Dachengqi Decoction Attenuates Inflammatory Response via Inhibiting HMGB1 Mediated NF-kappaB and P38 MAPK Signaling Pathways in Severe Acute Pancreatitis. Cellular physiology and biochemistry : international journal of experimental cellular physiology, biochemistry, and pharmacology 2015;37:1379-1389.

24 Kang R, Zhang Q Hou W, Yan Z, Chen R, Bonaroti J, Bansal P, Billiar TR, Tsung A, Wang Q, Bartlett DL, Whitcomb DC, Chang EB, Zhu X, Wang H, Lu B, Tracey KJ, Cao L, Fan XG, Lotze MT, Zeh HJ, 3rd, Tang D: Intracellular Hmgb1 inhibits inflammatory nucleosome release and limits acute pancreatitis in mice. Gastroenterology 2014;146:1097-1107.

25 Chen M, Liu Y, Varley P, Chang Y, He XX, Huang H, Tang D, Lotze MT, Lin J, Tsung A: High-Mobility Group Box 1 Promotes Hepatocellular Carcinoma Progression through miR-21-Mediated Matrix Metalloproteinase Activity. Cancer research 2015;75:1645-1656.

-26 Luan ZG, Zhang XJ, Yin XH, Ma XC, Zhang H, Zhang C, Guo RX: Downregulation of HMGB1 protects against 


\section{Cellular Physiology Cell Physiol Biochem 2018;51:2198-2211

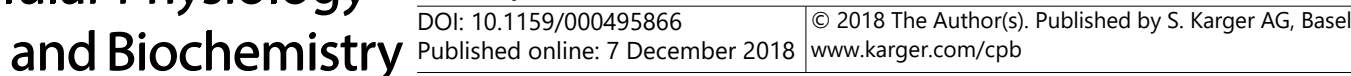 \\ Published onlıne: / December 2018 www.karger.com/cpb}

the development of acute lung injury after severe acute pancreatitis. Immunobiology 2013;218:1261-1270.

27 Luan ZG, Zhang J, Yin XH, Ma XC, Guo RX: Ethyl pyruvate significantly inhibits tumour necrosis factoralpha, interleukin-1beta and high mobility group box 1 releasing and attenuates sodium taurocholateinduced severe acute pancreatitis associated with acute lung injury. Clinical and experimental immunology 2013;172:417-426.

28 Ma P, Yu K, Yu J, Wang W, Ding Y, Chen C, Chen X, Zhao K, Zuo T, He X, Shi Q Ren J: Effects of Nicotine and Vagus Nerve in Severe Acute Pancreatitis-Associated Lung Injury in Rats. Pancreas 2016;45:552-560.

29 Jo IJ, Bae GS, Park KC, Choi SB, Jung WS, Jung SY, Cho JH, Choi MO, Song HJ, Park SJ: Scolopendra subspinipes mutilans protected the cerulein-induced acute pancreatitis by inhibiting high-mobility group box protein-1. World journal of gastroenterology 2013;19:1551-1562.

-30 Jensen JN, Cameron E, Garay MV, Starkey TW, Gianani R, Jensen J: Recapitulation of elements of embryonic development in adult mouse pancreatic regeneration. Gastroenterology 2005;128:728-741.

31 Cheng BQ, Liu CT, Li WJ, Fan W, Zhong N, Zhang Y, Jia XQ, Zhang SZ: Ethyl pyruvate improves survival and ameliorates distant organ injury in rats with severe acute pancreatitis. Pancreas 2007;35:256-261.

-32 Sawa H, Ueda T, Takeyama Y, Yasuda T, Shinzeki M, Nakajima T, Kuroda Y: Blockade of high mobility group box-1 protein attenuates experimental severe acute pancreatitis. World journal of gastroenterology 2006;12:7666-7670.

33 Yuan H, Jin X, Sun J, Li F, Feng Q, Zhang C, Cao Y, Wang Y: Protective effect of HMGB1 a box on organ injury of acute pancreatitis in mice. Pancreas 2009;38:143-148. 